\title{
ON THE CARLESON MEASURE CRITERION IN LINEAR SYSTEMS THEORY
}

\author{
BERNHARD H. HAAK
}

\begin{abstract}
In Ho, Russell [15, and Weiss 33, a Carleson measure criterion for admissibility of one-dimensional input elements with respect to diagonal semigroups is given. We extend their results from the Hilbert space situation $X=\ell_{2}$ and $L^{2}$-admissibility to the more general situation of $L^{p}$-admissibility on $\ell_{q}$-spaces. In case of analytic diagonal semigroups we present a new result that does not rely on Laplace transform methods. A comparison of both criteria leads to result of $L^{p}$-admissibility for reciprocal systems in the sense of Curtain [5].
\end{abstract}

\section{INTRODUCTION}

Consider the infinite dimensional linear system described by the differential equation

$$
\begin{cases}x^{\prime}(t)+A x(t) & =B u(t) \\ x(0) & =x_{0} \in X\end{cases}
$$

on a Banach space $X$. We assume that $-A$ generates a strongly continuous semigroup $S(\cdot)$ on $X$. Then the spectrum of $A$ is necessarily contained in some half plane $\mathbb{C}_{\beta}, \beta \in \mathbb{R}$. Choosing some element $\lambda$ of the resolvent set $\varrho(-A)$, we may define the Banach space $X_{1}=(\mathcal{D}(A),\|(\lambda+A) \cdot\|)$, and the so-called extrapolation space $X_{-1}$ defined as the completion of $X$ with respect to a resolvent-norm $\left\|(\lambda+A)^{-1} \cdot\right\|$ for some fixed $\lambda \in \varrho(-A)$ (see [8, 14] for more details on these spaces). Since $X$ is dense in $X_{-1}$, the semigroup $S$ extends in a natural way to $X_{-1}$; for the sake of simplicity we denote this extension also $S$.

Let $U$ be another Banach space, and assume that $B \in \mathcal{B}\left(U, X_{-1}\right)$. A solution of (1.1) is necessarily of the form

$$
x(t)=S(t) x_{0}+\int_{0}^{t} S(t-s) B u(s) d s
$$

Notice that $x(t)$ is a well-defined element of $X_{-1}$ for $t \geq 0$ but that, in general, there is no reason why $x(t)$ should be an element of $X$.

Definition 1.1. Let $p \in[1, \infty]$. A bounded operator $B \in \mathcal{B}\left(U, X_{-1}\right)$ is called finite-time $L^{p}$-admissible for $A$, if for every $\tau>0$ there exists a constant $K>0$ such that for all $u \in L^{p}([0, \tau], U)$

$$
\left\|\int_{0}^{t} S(t-s) B u(s) d s\right\|_{X} \leq K\|u\|_{L^{p}(0, \tau ; U)} \quad t \in[0, \tau] .
$$

The integral here is considered as a Bochner integral in $X_{-1}$ and we suppose that the integral takes values in $X$. The operator $B$ is called (infinite-time) $L^{p}$-admissible for $A$, if the constant $K$ in (1.2) can be chosen independently of $\tau>0$.

Date: October 30, 2018.

1991 Mathematics Subject Classification. 93C05, 93B28,47D06,42B25.

Key words and phrases. Linear Systems Theory, Admissibility, Carleson Measures, Reciprocal Systems. 
In the case where the rank one operator $B$, defined by $B u(t)=b u(t)$ for $b \in X_{-1}$, is (finite-time) $L^{p}$-admissible, we say that $b$ is an $L^{p}$-admissible input element. When $p<\infty$, a density argument shows that $B$ is (infinite-time) $L^{p}$-admissible if and only if the estimate

$$
\left\|\int_{0}^{\infty} S(s) B u(s) d s\right\|_{X} \leq K\|u\|_{L^{p}(0, \infty ; U)}
$$

holds for all $u$ in the Schwartz class. We remark that due to the semigroup property, $L^{p}$-admissibility in finite and infinite time coincide for uniformly exponentially stable semigroups. For the special case $p=2$ there is a large literature on the notion of admissibility. Among the early abstract formulations of admissibility we refer e.g. to [25, 34, 35, 36. A more recent publication [17] gives a detailed survey of the subject.

In this article we restrict our focus to sequence spaces $X=\ell_{q}$. Moreover, we assume $-A$ to be the generator of a bounded diagonal semigroup $S(\cdot)$ where $(S(t) x)_{n}=\exp \left(-\lambda_{n} t\right) x_{n}, n \in \mathbb{N}$. We consider the control system

$$
x^{\prime}(t)+A x(t)=b u(t), \quad \text { for } t>0 \quad \text { and } \quad x(0)=0
$$

with input element $b=\left(b_{n}\right) \in X_{-1}:=\left\{\left(\xi_{n}\right):\left(\frac{\xi_{n}}{1+\lambda_{n}}\right) \in X\right\}$.

Our aim is to give conditions for $L^{p}$-admissibility of $b$ for $A$ on $X=\ell_{q}$, i.e. conditions to guarantee the estimate

$$
\left(\sum_{n=1}^{\infty}\left|b_{n}\right|^{q}\left|\int_{0}^{\infty} e^{-\lambda_{n} s} u(s) d s\right|^{q}\right)^{1 / q} \leq K\|u\|_{p}
$$

or the respective estimate for convolutions derived from (1.2) with a uniform constant $K$ for all times $t>0$. We present two criteria, both via a (generalised) Carleson measure property of a discrete measure, associated with the numbers $b_{n}$ and the eigenvalues $\lambda_{n}$ of $A$.

The first result is a generalisation of a result of Ho, Russell and WeIss. It provides a sufficient condition for $L^{p}$-admissibility in the case $p \leq 2$ that characterises $L^{p}$-admissibility if in addition $p^{\prime} \leq q$ where $p^{\prime}$ is the conjugate exponent defined by $1 / p+1 / p^{\prime}=1$. The second result is new and treats the case of analytic semigroups. It does not rely on Laplace transform methods and therefore allows $p$ and $q$ to be chosen freely in $(1, \infty)$. It provides a sufficient condition for all $p$ and $q$ that is necessary in the case $p \leq q$. Notice that, in contrast with the first result this allows us to characterise $L^{p}$-admissibility on $X=\ell_{p}$ for all $p \in(1, \infty)$.

In the last section we discuss the two criteria. This leads naturally to so-called 'reciprocal systems' and the question of whether admissibility of a system implies (or is implied by) admissibility of the associated reciprocal system. We present two results, one for general strongly continuous semigroups and the other for analytic semigroups.

\section{Preliminaries on $\alpha$-CARleson measures}

Let $\mathbb{R}_{+}^{d+1}:=\mathbb{R}^{d} \times(0, \infty)$. Let $P_{t}(x)=c_{d} t\left(t^{2}+\|x\|^{2}\right)^{-\frac{d+1}{2}}$ be the Poisson kernel on $\mathbb{R}^{d}$ and let $(\mathscr{P} f)(x, t)=\int P_{t}(x-y) f(y) d y$ be the Poisson extension of $f$ to the half space $\mathbb{R}_{+}^{d+1}$. For $x \in \mathbb{R}^{d}$, let $\Gamma(x)$ denote the cone $\left\{(y, t) \in \mathbb{R}_{+}^{d+1}:\|x-y\|<t\right\}$. Given an open set $O \subseteq \mathbb{R}^{d}$ let $T(O)$ denote the "tent"

$$
T(O):=\left(\bigcup_{x \notin O} \Gamma(x)\right)^{\complement} .
$$


If $d=1$ and $O$ is an open interval, this can be visualised as an isosceles triangle with base $O$ in the half plane. Let $\mathcal{B} o\left(\mathbb{R}_{+}^{d+1}\right)$ be the set of all non-negative Borel-measures on $\mathbb{R}_{+}^{d+1}$.

Definition 2.1 (embedding $\alpha$-Carleson measures). Let $\alpha>0$ and $q \in[1, \infty)$ such that $\alpha q>1$. A non-negative measure $\mu \in \mathcal{B} o\left(\mathbb{R}_{+}^{d+1}\right)$ satisfying

$$
\|f\|_{L^{q}\left(\mathbb{R}_{+}^{d+1}, \mu\right)} \leq M_{q}\|f\|_{H^{\alpha q}\left(\mathbb{R}_{+}^{d+1}\right)}
$$

for all $f \in H^{\alpha q}\left(\mathbb{R}_{+}^{d+1}\right)$ is called an embedding $\alpha$-Carleson measure.

The above definition is independent of $q$; indeed, if $f \in L^{\alpha p}\left(\mathbb{R}^{d}\right)$, then its Poisson extension $\left|P_{t} * f\right|^{p / q}$ is subharmonic and therefore, $\left|P_{t} * f\right|^{p / q} \leq P_{t} *|f|^{p / q}$. This implies that

$$
\left\|P_{t} * f\right\|_{L^{p}(\mu)}^{p} \leq\left\|P_{t} *|f|^{p / q}\right\|_{L^{q}(\mu)}^{q} \leq C\left\||f|^{p / q}\right\|_{L^{\alpha q}\left(\mathbb{R}^{d}\right)}^{q}=\|f\|_{L^{\alpha p}}^{p} .
$$

In case $\alpha=1$ we simply speak of Carleson measures. Recall that $u \in H^{q}\left(\mathbb{R}_{+}^{d+1}\right)$ if, and only if $u=\mathscr{P} f$ for some $f \in L^{q}\left(\mathbb{R}^{d}\right)$ (see, e.g. [26, III.4.2]) and that $\|\mathscr{P} f\|_{H^{q}}=\|f\|_{L^{q}}$.

Definition 2.2 (geometric $\alpha$-Carleson measures). A non-negative Borel measure $\mu \in \mathcal{B} o\left(\mathbb{R}_{+}^{d+1}\right)$ is called geometric $\alpha$-Carleson if

$$
\mu(T(Q))^{\alpha} \leq c|Q|
$$

for all cubes $Q \subseteq \mathbb{R}^{d}$.

In the literature the choice of the exponent is not consistent (sometimes $\alpha$ is replaced by $1 / \alpha$ ). For all $\alpha>0$, embedding $\alpha$-Carleson measures are also geometric $\alpha$-Carleson; this can be seen by applying the embedding estimate to the function $P_{t} * f$ where $f=\mathbb{1}_{Q}$ is the characteristic function of $Q$. A celebrated theorem of CARleson [3, 4] states that both notions coincide for $\alpha=1$, DUREN [7] extended this result to $\alpha \in(0,1]$. In case $\alpha>1$, the embedding $\alpha$-Carleson property is strictly stronger than geometric one as TAYLOR and WILLIAMS 28 show with a counterexample on the complex unit disk. In Example 2.5 (d) below we give a similar example on the half space $\mathbb{R}_{+}^{2}$. Both notions were subsequently treated in AmAR and BonAmi 2]. Variants of these results with weighted Hardy spaces have been obtained by MCPHAIL 22 and NAKAZI 23. Applications of Carleson measures in the context of systems theory can be found e.g. in recent publications in [16] and 37.

Let $L^{\alpha, 1}\left(\mathbb{R}_{+}^{d+1}\right)$ denote the smallest Lorentz space of $\alpha$-integrable functions. Then $L^{\alpha, 1}\left(\mathbb{R}_{+}^{d+1}\right) \subsetneq L^{\alpha, \alpha}\left(\mathbb{R}_{+}^{d+1}\right)=L^{\alpha}\left(\mathbb{R}_{+}^{d+1}\right)$ for $\alpha>1$. If, in place of (2.1), one merely knows that $\|\mathscr{P} f\|_{L^{1}\left(\mathbb{R}_{+}^{d+1}, \mu\right)} \leq C\|f\|_{L^{\alpha, 1}\left(\mathbb{R}_{+}^{d+1}\right)}, \mu$ is called weakly embedding $\alpha$-Carleson. We shall not go into details about this notion, but just mention that it is linked to the distinction between requiring the estimate (2.2) on all cubes or on all open sets (see [2, Theorem 1]), and consequently, the weak embedding notion implies the geometric one. In case $\alpha \leq 1$ all notions of $(\alpha)$-Carleson coincide by the Carleson-Duren result; all three are strictly different in case $\alpha>1$.

In the case $d=1$, VIDENSII 31 gives several equivalent conditions for $\mu$ to be embedding $\alpha$-Carleson for $\alpha>1$, one of which is the Fefferman-Stein maximal function description. It follows from a maximal inequality due to FEFFERMAN and STEIN 9. Indeed, let

$$
\psi_{\mu}(x):=\sup \left\{\frac{\mu(T(Q))}{|Q|}: x \in Q\right\}
$$

where the sets $Q$ in the above supremum are cubes in $\mathbb{R}^{d}$ containing the element $x$. We call $\psi_{\mu}$ the Fefferman-Stein maximal function. 
Theorem 2.3. Let $\alpha>1$ and let $\beta$ be its conjugate exponent satisfying $1 / \alpha+1 / \beta=1$. Then $\mu \in \mathcal{B} o\left(\mathbb{R}_{+}^{d+1}\right)$ is embedding $\alpha$-Carleson if and only if $\psi_{\mu} \in L^{\beta}\left(\mathbb{R}^{d}\right)$.

Proof. Suppose that $\psi_{\mu} \in L^{\beta}\left(\mathbb{R}^{d}\right)$. The Fefferman and Stein inequality [9, Theorem 2] reads: $\|\mathscr{P} f\|_{L^{p}(\mu)} \leq\|f\|_{L^{p}\left(\psi_{\mu}(x) d x\right)}$. Since $\psi_{\mu} \in L^{\beta}$, Hölder's inequality now implies that $\mu$ is embedding $\alpha$-Carleson.

Conversely, if $\mu$ is embedding $\alpha$-Carleson, $P_{t} *: L^{\alpha} \rightarrow L^{1}(\mu)$ is bounded, which is equivalent to $F \in L^{\beta}\left(\mathbb{R}^{d}\right)$ where $F(y):=\int P_{t}(x-y) d \mu(x, t)$ is the 'balayée' of $\mu$. Notice that $(x, t) \in T(Q)$ implies that $B(x, t) \subseteq Q$ and so

$$
\int_{Q} P_{t}(x-y) d y \geq \int_{B(x, t)} P_{t}(x-y) d y=\int_{B(0,1)} P_{1}(y) d y=: \varepsilon_{d}>0
$$

for $(x, t) \in T(Q)$. Consequently,

$$
\frac{1}{|Q|} \int_{Q} F(y) d y=\frac{1}{|Q|} \int_{T(Q)} \int_{Q} P_{t}(x-y) d y d \mu(x, t) \geq \varepsilon_{d} \frac{\mu(T(Q)}{|Q|} .
$$

Denoting by $M F$ the (uncentred) maximal function of $F$ the above estimates yields $(M F)(x) \geq \varepsilon_{d} \psi_{\mu}(x)$ and therefore $\psi_{\mu} \in L^{\beta}\left(\mathbb{R}^{d}\right)$.

Another way of verifying that $\mu$ is embedding $\alpha$-Carleson is to show that (2.1) holds for linear combinations of reproducing kernels by a density argument. Indeed, a continuous linear functional $l \in\left(H^{p}\left(\mathbb{R}^{d}\right)\right)^{\prime}=H^{p^{\prime}}\left(\mathbb{R}^{d}\right)$ that vanishes on all reproducing kernels $k_{x}$ satisfies $0=l\left(k_{x}\right)=l(x)$ and therefore $l=0$. It is natural to ask whether the embedding $\alpha$-Carleson property can be tested on the reproducing kernels without taking linear combinations. This is sometimes referred to as the 'reproducing kernel thesis'.

Lemma 2.4. Assume that for $p, q \in(1, \infty)$ there exists a constant $M>0$ such that

$$
\left\|k_{z}\right\|_{\left.L^{q}\left(\mathbb{R}_{+}^{2}\right), \mu\right)} \leq M\left\|k_{z}\right\|_{H^{p}\left(\mathbb{R}_{+}^{2}\right)}
$$

for all $z \in \mathbb{C}_{+}$. Then $\mu$ is geometric $\alpha$-Carleson where $\alpha=p / q$.

Proof. Since $k_{\lambda}(z)=1 /(z+\bar{\lambda})$, letting $\operatorname{Re}(\lambda)=\xi>0$ the substitution $y=\xi t$ shows

$$
\left\|k_{\lambda}\right\|_{H^{p}}^{p}=\int_{-\infty}^{\infty} \frac{d y}{\left(y^{2}+\xi^{2}\right)^{p / 2}}=\int_{-\infty}^{\infty} \frac{\xi d t}{\xi^{p}\left(t^{2}+1\right)^{p / 2}}=C_{p}^{p} \xi^{1-p},
$$

and therefore, $\left\|k_{\lambda}\right\|_{H^{p}}=C_{p}(\operatorname{Re}(\lambda))^{-1 / p^{\prime}}$. For $\omega \in \mathbb{R}$ and $r>0$, let $\lambda=i \omega+r$ and use the shorter notation $T_{\omega, r}$ for the tent $T((\omega-r, \omega+r))$. Then the triangle inequality yields $\left|k_{\lambda}(z)\right| \geq 1 /(2 r)$ for all $z \in T(\omega-r, \omega+r)$ and therefore,

$$
\begin{aligned}
\mu\left(T_{\omega, r}\right) & =\int_{T_{\omega, r}} d \mu \leq(2 r)^{q} \int_{T_{\omega, r}}\left|k_{\lambda}(z)\right|^{q} d \mu \\
& \leq M(2 r)^{q}\left\|k_{\lambda}(z)\right\|_{H^{p}}^{q}=M C_{p} 2^{q} r^{q-\frac{q}{p^{\prime}}=C r^{1 / \alpha}}
\end{aligned}
$$

This shows that $\mu$ is geometric $\alpha$-Carleson as claimed.

Using the Lemma and the results from Duren-Carleson and the aforementioned counterexample from Taylor and Williams, it becomes clear that a $H^{p}-L^{q}(\mu)$ version of the reproducing kernel thesis holds if, and only if $p \leq q$.

Examples 2.5 . Let $d=1$ and identify $\mathbb{R}_{+}^{2}$ with the right half plane $\mathbb{C}_{+}$.

(a) The Lebesgue measure in $\mathbb{R}_{+}^{2}$ is clearly geometric $1 / 2$-Carleson and therefore also embedding $1 / 2-$ Carleson. 
(b) For $\omega \in(0, \pi)$, let $S(\omega):=\{z \in \mathbb{C} \backslash\{0\}:|\arg z|<\omega\}$ and let $S(0):=(0, \infty)$. We say that $\mu$ is sectorial, if the support of $\mu$ is contained in a finite union of sectors $i \omega_{k}+S\left(\theta_{k}\right), k=1 \ldots, n$ with $\omega_{k} \in \mathbb{R}$ and $\theta \in(0, \pi / 2)$.

If $\mu$ is sectorial and geometric $\alpha$-Carleson, intervals $I$ containing a point $x$ with sufficiently large absolute value need a length of at least $\varepsilon|x|$ for $T(I)$ to intersect with the support of $\mu$. Therefore, geometric $\alpha$-Carleson implies that the Fefferman-Stein maximal function is weak $-L^{\beta}$. Consequently, sectorial measures $\mu$ that are geometric $\alpha$ - and geometric $\widetilde{\alpha}-$ Carleson are embedding $\gamma$-Carleson for all $\gamma \in(\alpha, \widetilde{\alpha})$ by Marcinkiewicz' interpolation theorem.

(c) Sectorial measures $\mu$ are embedding $\alpha$-Carleson if and only if the function $g$ defined by $g(r):=\frac{1}{r} \mu(\{\operatorname{Re}(z)<r\})$ satisfies $g \in L^{\beta}\left(\mathbb{R}_{+}\right)$(see [31, Remark, p. 188]).

(d) For $\alpha>1$, measures that are geometric- but not embedding $\alpha$-Carleson are given in 28 for the unit disc. A simplified half-space version reads as follows: let $\alpha \in(1,2)$ and let $\mu_{\alpha}$ be the measure $\mu_{\alpha}=\left(\frac{x}{1+x}\right)^{1 / \alpha-1} d x$, supported on the real line. Then, for every interval $I$, one has $\mu_{\alpha}(T(I)) \leq$ $C|I|^{1 / \alpha}$, whence $\mu_{\alpha}$ is geometric $\alpha$-Carleson. For a real parameter $r>0$ let $F(z)=(r+z)^{-2 / p}$. For $p, q \in(1, \infty)$ with $\alpha=p / q$ one has

$$
\|F\|_{H^{p}}=\left(\int_{-\infty}^{\infty} \frac{1}{r^{2}+t^{2}} d t\right)^{1 / p}=c_{p} r^{-1 / p}
$$

whereas a substitution yields

$$
\|F\|_{L^{q}\left(\mu_{\alpha}\right)}=r^{-1 / q}\left(\int_{0}^{\infty}\left(\frac{t}{1+r t}\right)^{q / p-1} \frac{1}{(1+t)^{2 q / p}} d t\right)^{1 / q} .
$$

By Lebesgue's dominated convergence theorem, one has $\|F\|_{L^{q}\left(\mu_{\alpha}\right)} \sim r^{-1 / q}$ for $r \rightarrow 0+$. Therefore, the embedding $\alpha$-Carleson property fails.

(e) Finally we give an example of a discrete measure that is geometric $\alpha-$ Carleson without being embedding $\alpha$-Carleson: let $\varepsilon \in[0,1)$ and $\gamma \geq 1$ such that $\alpha=\frac{\gamma}{1-\varepsilon}>1$. Let $\mu=\sum_{n \in \mathbb{Z}^{*}}|n|^{-\varepsilon} \delta_{\lambda_{n}}$ where $\lambda_{n}=1+i n^{\gamma}$. Since $\mu(T(B(x, r)))=0$ for $r<1$ we may suppose $r \geq 1$. Since $\gamma \geq 1$ the support of $\mu$ is either equidistant or thins out at infinity. Moreover weights decrease as $|n|$ increases. Therefore

$$
\begin{aligned}
\mu(T(B(x, r))) & \leq \mu(T(B(0,2 r))) \leq 2 \sum_{j=0}^{\left\lfloor(2 r-1)^{1 / \gamma}\right\rfloor} j^{-\varepsilon} \\
& \leq 2 \int_{1}^{(2 r)^{1 / \gamma}}(t-1)^{-\varepsilon} d t \leq \frac{2}{1-\varepsilon}(2 r)^{\frac{1-\varepsilon}{\gamma}},
\end{aligned}
$$

whence $\mu$ is geometric $\alpha$-Carleson. Considering the tents $T((-x-1, x+1))$ for $x>1$, however, yields the estimate

$$
\psi_{\mu}(x) \geq c \frac{\left(\left\lfloor(x)^{\frac{1}{\gamma}}\right\rfloor^{1-\varepsilon}-1\right)}{x} .
$$

Let $\beta$ denote the dual exponent of $\alpha$. Then, for $n \geq 2$, it readily follows that

$$
\int_{n^{\gamma}}^{(n+1)^{\gamma}}\left|\psi_{\mu}(s)\right|^{\beta} d s \geq \frac{c_{\varepsilon, \beta, \gamma}}{n+1}
$$

where $c_{\varepsilon, \beta, \gamma}>0$. Now Theorem 2.3 allows us to conclude that $\mu$ cannot be embedding $\alpha$-Carleson since the above estimate shows $\psi_{\mu} \notin L^{\beta}(\mathbb{R})$. 
In order to apply Carleson-type criteria for admissibility, we wish to replace the Poisson kernel by more general convolution kernels. In case $\alpha \leq 1$ the following result is due to STEIN [27, Theorem II.5.9].

Theorem 2.6. Let $q \in(1, \infty)$ and $\alpha>1 / q$. Let $\Phi$ be a function on $\mathbb{R}^{d}$ that admits a radial, non-increasing, bounded and integrable dominating function $\varphi$. For $t>0$ put $\Phi_{t}(x):=t^{-d} \Phi(x / t)$. Then for all embedding $\alpha$-Carleson measures $\mu$ on $\mathbb{R}_{+}^{d+1}$ the estimate

$$
\left(\int_{\mathbb{R}_{+}^{d+1}}\left|\left(\Phi_{t} * f\right)(x)\right|^{q} d \mu(t, x)\right)^{1 / q} \leq c\|f\|_{L^{\alpha q}\left(\mathbb{R}^{d}\right)}
$$

holds for all $f \in L^{\alpha q}\left(\mathbb{R}^{d}\right)$.

Proof. Notice that if $F(x, t):=\left(\Phi_{t} * f\right)(x)$ and $F_{\Phi}^{*}(x):=\sup _{|x-y|<t}|F(y, t)|$, then $s>0$

$$
\left\{(y, t) \in \mathbb{R}_{+}^{d+1}:|F(y, t)|>s\right\} \subseteq T\left(O_{s}\right)
$$

where $O_{s}:=\left\{x \in \mathbb{R}^{d}: F_{\Phi}^{*}(x)>s\right\}$, see, e.g., [27, Section II.2.3]. Now inequality (2.4) implies $\left(\mathscr{P} F_{\Phi}^{*}\right)(x, t) \geq s \varepsilon_{d}$ for $(x, t) \in T\left(O_{s}\right)$. The problem now boils down to the Poisson kernel estimate as follows:

$$
\begin{aligned}
\|F\|_{L^{q}(\mu)}^{q} & =q \int_{0}^{\infty} s^{q-1} \mu(|F|>s) d s \leq q \int_{0}^{\infty} s^{q-1} \mu\left(T\left(O_{s}\right)\right) d s \\
& \leq q \int_{0}^{\infty} s^{q-1} \mu\left(\left(\mathscr{P} F_{\Phi}^{*}\right)>s \varepsilon_{d}\right) d s=\varepsilon_{d}^{-q}\left\|\mathscr{P} F_{\Phi}^{*}\right\|_{L^{q}(\mu)}^{q} .
\end{aligned}
$$

The desired estimate

$$
\|F\|_{L^{q}(\mu)} \leq \frac{1}{\varepsilon_{d}}\left\|\mathscr{P} F_{\Phi}^{*}\right\|_{L^{q}(\mu)} \leq \frac{C}{\varepsilon_{d}}\left\|F_{\Phi}^{*}\right\|_{L^{\alpha q}\left(\mathbb{R}^{d}\right)} \leq \frac{C M}{\varepsilon_{d}}\|f\|_{L^{\alpha q}\left(\mathbb{R}^{d}\right)}
$$

follows from Theorem 2.3 and [27, Proposition II.2.1].

\section{CRiteria FOR ADMissibility OF DiAgONAL SYSTEMS}

As a first result we present a Carleson measure criterion for admissibility of diagonal systems.

Theorem 3.1. Let $q \in(1, \infty), p \in(1,2]$ and $\alpha q=p^{\prime}$ where $p^{\prime}$ is the dual exponent of $p$. On $X=\ell_{q}$ let $A$ be a diagonal operator with eigenvalues $\lambda_{n} \in \mathbb{C}_{+}$and let $b=\left(b_{n}\right)$ a sequence of complex numbers. Consider the discrete measure $\mu=$ $\sum_{n}\left|b_{n}\right|^{q} \delta_{\lambda_{n}}$

(a) If $\mu$ is an embedding $\alpha$-Carleson measure, then $b \in X_{-\theta}$ for all $\theta>1 / p^{\prime}$ and $b$ is an infinite-time $L^{p}$-admissible input element for $A$.

(b) If $b$ in an infinite-time $L^{p}$-admissible input element for $A$, then $b \in X_{-\theta}$ for all $\theta>1 / p^{\prime}$ and $\mu$ is geometric $\alpha$-Carleson.

In particular, the $\alpha$-Carleson property of $\mu$ characterises the $L^{p}$-admissibility of $b$ in the case $p^{\prime} \leq q$. Moreover, the result in $(b)$ is optimal in the sense that for $p^{\prime}>q$, the measure $\mu$ is not embedding $\alpha$-Carleson in general.

Proof. (a) First assume that $\mu$ is an embedding $\alpha$-Carleson measure. Then $\mu$ is also geometric $\alpha$-Carleson. To show $b \in X_{-\theta}$ we employ essentially the argument in [33, Proposition 3.1]: let $\Delta_{n}=T\left(\left[-2^{n}, 2^{n}\right]\right) \backslash T\left(\left[-2^{n-1}, 2^{n-1}\right]\right)$. Then

$$
\begin{aligned}
\sum_{k} \frac{\left|b_{k}\right|^{q}}{\left|1+\lambda_{k}\right|^{\theta q}} & \leq \sum_{n \in \mathbb{Z}} \frac{1}{\left(1+2^{n-1}\right)^{\theta q}} \sum_{\lambda_{k} \in \Delta_{n}}\left|b_{k}\right|^{q} \\
& \leq \sum_{n \in \mathbb{Z}} \frac{1}{\left(1+2^{n-1}\right)^{\theta q}} \mu\left(T\left(\left[-2^{n}, 2^{n}\right]\right)\right) \leq C \sum_{n \in \mathbb{Z}} \frac{2^{\frac{n q}{p}}}{1+2^{n \theta q}}
\end{aligned}
$$

which is finite for all $\theta>1 / p^{\prime}$. 
To show that $b$ is infinite-time $L^{p}$-admissible, let $\tau>0$ and let $u$ be a test function with support in $[0, \tau]$. Then its Laplace transform $\mathcal{L} u$ is bounded and analytic on the right half plane and therefore can be reproduced by its (non-tangential) boundary values on $i \mathbb{R}$. Notice that $(\mathcal{L} u)(i s)=\mathcal{F} u(s)$ where $\mathcal{F} u$ denotes the Fourier transform of $u$.

$$
\begin{aligned}
\left\|\int_{0}^{\infty} S(s) b u(s) d s\right\|_{\ell_{q}} & =\left(\sum_{n=0}^{\infty}\left|b_{n}\right|^{q}\left|\int_{0}^{\infty} e^{-\lambda_{n} s} u(s) d s\right|^{q}\right)^{1 / q} \\
\text { (definition of } \mu) & =\left(\int_{\mathbb{R}_{+}^{2}}|(\mathcal{L} u)(t+i x)|^{q} d \mu(x, t)\right)^{1 / q} \\
& =\left\|P_{t} *(\mathcal{F} u)\right\|_{L^{q}(\mu)} \leq C\|\mathcal{F} u\|_{L^{p^{\prime}}(\mathbb{R})} \\
& \leq C\|u\|_{L^{p}\left(\mathbb{R}_{+}\right)} .
\end{aligned}
$$

In the last estimate we make use of $p \leq 2$ and the boundedness of the Fourier transform from $L^{p}$ to $L^{p^{\prime}}$. Notice that the obtained constant is independent of $\tau>0$.

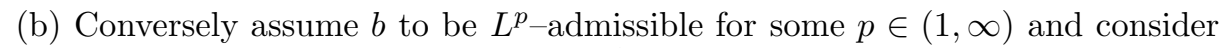
the reproducing kernel function $k_{z}(\lambda)=\frac{1}{\lambda+\bar{z}}$ for $\operatorname{Re}(z)>0$. Then

$$
\begin{aligned}
\left\|k_{z}\right\|_{L^{q}(\mu)} & =\left(\sum_{n=0}^{\infty}\left|\frac{b_{n}}{\lambda_{n}+\bar{z}}\right|^{q}\right)^{1 / q}=\left\|\int_{0}^{\infty} T(t) b e^{-t \bar{z}} d t\right\|_{\ell_{q}} \\
& \leq C\left\|e^{-\cdot \bar{z}}\right\|_{L^{p}\left(\mathbb{R}_{+}\right)}=C(\operatorname{Re}(z))^{-1 / p}=C^{\prime}\left\|k_{z}\right\|_{H^{p^{\prime}}\left(\mathbb{C}_{+}\right)} .
\end{aligned}
$$

So, by Lemma 2.4 $\mu$ is geometric $\alpha$-Carleson. Consequently, $b \in X_{-\theta}$ for all $\theta>1 / p^{\prime}$.

It remains to show that $\mu$ is not embedding $\alpha$-Carleson in general. We use Example 2.5] (e)] For a given $q \in(1, \infty)$ we choose $\varepsilon \in(0,1)$ such that $q \varepsilon>1$. Then $b=\left(b_{n}\right)$ with $b_{n}=n^{-\varepsilon}$ will satisfy $b \in \ell_{q}$. For a given $\alpha>1$, let $\gamma$ and $\left(\lambda_{n}\right)$ be chosen as in the example. Let $p^{\prime}=\alpha q$. Using Hölder's inequality and the fact that $\operatorname{Re}\left(\lambda_{n}\right)=1$,

$$
\begin{aligned}
\left\|\int_{0}^{\infty} S(t) b u(t) d t\right\|_{\ell_{q}} & =\left(\sum_{n=1}^{\infty}\left|b_{n}\right|^{q}\left|\int_{0}^{\infty} e^{-\lambda_{n} t} u(t) d t\right|^{q}\right)^{1 / q} \\
& \leq\|u\|_{L^{p}\left(\mathbb{R}_{+}\right)}\left(\sum_{n=1}^{\infty}\left|b_{n}\right|^{q}\left\|e^{-t}\right\|_{L^{p^{\prime}\left(\mathbb{R}_{+}\right)}}^{q}\right)^{1 / q}
\end{aligned}
$$

and so $b$ is $L^{p}$ admissible. However, as shown in the Example 2.5 (e), the embedding $\alpha$-Carleson property fails.

Bibliographical notes. Part (a) of the theorem in the case $p=q=2$ is due to Ho, Russell 15] and the case $\alpha \leq 1$, in which the geometric and the embedding $\alpha-$ Carleson properties coincide, was found by Unteregge [30] independently. Part (b) of the theorem is due to WEIss 33, but we provide a different proof.

In the proof of Theorem 3.1, the embedding $\alpha$-Carleson property is applied somehow indirectly: first the desired estimate is transformed into an interpolation problem for the Laplace transform of $u$, which makes it necessary to use boundedness of the (inverse) Fourier transform; in the end the unaesthetic restriction $p \leq 2$ seems inevitable. This is particularly embarrassing since $p=q$ is a very natural case to consider. 
The next result overcomes this obstacle; it makes no use of the Laplace transform but instead directly uses the convolution estimate from Theorem 2.6. However, it requires analyticity of the semigroup $S(\cdot)$ as an additional assumption. We recall some notation: we recall that a sectorial operator $A$ of type $\omega \in[0, \pi)$ in a Banach space $X$ is a closed linear operator $A$ satisfying $\sigma(A) \subseteq \overline{S(\omega)}$ and, for any $\nu \in(\omega, \pi)$,

$$
\sup \{\|\lambda R(\lambda, A)\|:|\arg \lambda| \geq \nu\}<\infty .
$$

An operator $-A$ generates a bounded analytic semigroup in $X$ if and only if $A$ is a densely defined sectorial operator in $X$ of type $<\pi / 2$.

Theorem 3.2. Let $p, q \in(1, \infty)$ and $\alpha q=p$. Let $\theta \in(0, \pi / 2)$ and let $A$ be an injective diagonal operator on $X=\ell_{q}$ with eigenvalues $\lambda_{n} \in S(\theta)$. Let $b=\left(b_{n}\right)$ be $a$ sequence of complex numbers and consider the discrete measure $\mu=\sum_{n}\left|\frac{b_{n}}{\lambda_{n}}\right|^{q} \delta_{\lambda_{n}^{-1}}$.

(a) If $\mu$ is embedding $\alpha$-Carleson, then $b \in X_{-\theta}$ for all $\theta>1 / p^{\prime}$ and $b$ is an (infinite-time) $L^{p}$-admissible input element for $A$.

(b) If $b$ is infinite-time $L^{p}$-admissible for $A$, then $b \in X_{-\theta}$ for all $\theta>1 / p^{\prime}$ and $\mu$ is geometric $\alpha$-Carleson.

In particular, the $\alpha$-Carleson property of $\mu$ characterises $L^{p}$-admissibility of $b$ in the case $p \leq q$.

Notice that if $0 \in \varrho(A)$, then $\left(\frac{b_{n}}{\lambda_{n}}\right)_{n} \in \ell_{q}$ and consequently $\mu$ is a finite measure.

Proof. (a) Let $\mu$ be an embedding $\alpha$-Carleson measure. To see that $b \in X_{-\theta}$ for all $\theta>1 / p^{\prime}$ is essentially the same as in the proof of Theorem 3.1 and so we omit it. To show infinite-time admissibility, choose an interval $[0, \tau]$ with $\tau>0$ and let $u \in L^{p}(0, \tau)$. We shall prove (1.2) with a constant $K$ that is independent of $\tau>0$.

Let $r_{n}=\operatorname{Re}\left(\lambda_{n}\right)$ and let $\widetilde{\mu}=\sum_{n}\left|\frac{b_{n}}{\lambda_{n}}\right|^{q} \delta_{\left(r_{n}^{-1}+i \tau\right)}$. We claim that $\widetilde{\mu}$ is an embedding $\alpha$-Carleson-measure on $\mathbb{R}_{+}^{2}$ as well. This has nothing to do with $\tau$ and so it is sufficient to show that $\mu$ is an embedding $\alpha$-Carleson measure on $\mathbb{R}_{+}^{2}$ if and only if $\nu=\sum_{n}\left|\frac{b_{n}}{r_{n}}\right|^{q} \delta_{r_{n}^{-1}}$ is an embedding $\alpha$-Carleson measure. To prove this claim we use the shorter notation $T_{\omega, r}$ for the tent $T((\omega-r, \omega+r))$. A simple geometric consideration, along with the fact that on the sector $S(\theta)$, real parts and absolute values are equivalent up to a constant of $\frac{1}{\cos (\theta)}$, shows that

$$
\mu\left(T_{w, r}\right) \leq \nu\left(T_{0, r}\right) \leq \frac{1}{\cos (\theta)^{q}} \mu\left(T_{0,|w|+r}\right) .
$$

If $\alpha \leq 1$ and if $\nu$ is $\alpha$-Carleson, the above inequality immediately yields that $\mu$ is $\alpha$-Carleson. Next, suppose that $\alpha \leq 1$ and that $\mu$ is $\alpha$-Carleson. Observe, that we may suppose for any tent $T_{\omega, r}$ that $|\omega| \leq \tan (\theta) r$ since otherwise there is no intersection of the tent with the sector $S(\theta)$. This implies the estimate

$$
\nu\left(T_{\omega, r}\right) \leq \nu\left(T_{0, r}\right) \leq \frac{1}{\cos (\theta)^{q}} \mu\left(T_{0,|w|+r}\right) \leq \frac{C}{\cos (\theta)^{q}}(|\omega|+r)^{1 / \alpha} \leq \frac{C(1+\tan (\theta))}{\cos (\theta)^{q}} r^{1 / \alpha}
$$

and so $\nu$ is $\alpha$-Carleson. Finally, if $\alpha>1$, the measures $\mu$ and $\nu$ are simultaneously embedding $\alpha$-Carleson by Example 2.5) (c) This proves the claim.

We now know that $\tilde{\mu}$ is an embedding $\alpha$-Carleson measure. Consider the kernel function

$$
\Phi(x):=\exp (-x) \mathbb{1}_{[0, \infty)}(x)
$$

which satisfies the assumptions of Theorem 2.6 (a radially decreasing integrable majorant is $\exp (-|x|))$. Notice that for $s>0, \Phi_{s}(x)=s^{-1} \exp (-x / s) \mathbb{1}_{[0, \infty)}(x)$. Then

$$
\left\|\int_{0}^{\tau} S(s) b u(\tau-s) d s\right\|_{\ell_{q}}=\left(\sum_{n=0}^{\infty}\left|\frac{b_{n}}{\lambda_{n}}\right|^{q}\left|\int_{0}^{\tau} \lambda_{n} e^{-\lambda_{n} s} u(\tau-s) d s\right|^{q}\right)^{1 / q}
$$




$$
\begin{aligned}
& \leq\left(\sum_{n=0}^{\infty}\left|\frac{b_{n}}{\lambda_{n}}\right|^{q}\left(\int_{0}^{\tau}\left|\frac{\lambda_{n}}{r_{n}}\right| r_{n} e^{-r_{n} s}|u(\tau-s)| d s\right)^{q}\right)^{1 / q} \\
& \leq \frac{1}{\cos (\theta)}\left(\sum_{n=0}^{\infty}\left|\frac{b_{n}}{\lambda_{n}}\right|^{q}\left(\left(\Phi_{r_{n}^{-1}} *|u|\right)(\tau)\right)^{q}\right)^{1 / q} \\
(\text { definition of } \widetilde{\mu}) & =\frac{1}{\cos (\theta)}\left(\int_{\mathbb{R}_{+}^{2}}\left(\left(\Phi_{s} *|u|\right)(t)\right)^{q} d \widetilde{\mu}(s, t)\right)^{1 / q} \\
& \leq \frac{c}{\cos (\theta)}\|u\|_{L^{p}}
\end{aligned}
$$

where we used Theorem 2.6 in the last estimate. Since the estimate is independent of the choice of $\tau>0, b$ is infinite-time admissible for $A$.

(b) Now assume that $b$ is $L^{p}$-admissible. Then, for $\operatorname{Re}(z)>0$, the reproducing kernel functions $k_{z}(\lambda)=\frac{1}{\lambda+\bar{z}}$ satisfy

$$
\begin{aligned}
\left\|k_{z}\right\|_{L^{q}(\mu)} & =\left(\sum_{n=0}^{\infty}\left|\frac{b_{n}}{\lambda_{n}} \frac{1}{\lambda_{n}^{-1}+\bar{z}}\right|^{q}\right)^{1 / q}=\frac{1}{|z|}\left(\sum_{n=0}^{\infty}\left|\frac{b_{n}}{\lambda_{n}+\bar{z}^{-1}}\right|^{q}\right)^{1 / q} \\
& =\frac{1}{|z|}\left\|\int_{0}^{\infty} T(t) b e^{-t \bar{z}^{-1}} d t\right\|_{\ell_{q}} \leq \frac{C}{|z|}\left\|\exp \left(-t \bar{z}^{-1}\right)\right\|_{L^{p}\left(\mathbb{R}_{+}\right)} \\
& =\frac{C}{|z|}\left(\frac{|z|^{2}}{\operatorname{Re}(z)}\right)^{1 / p} \leq C \cos (\theta)^{-2 / p}|\operatorname{Re}(z)|^{-1 / p^{\prime}} \\
& =C \cos (\theta)^{-2 / p}\left\|k_{z}\right\|_{H^{p}\left(\mathbb{R}_{+}^{2}\right)}
\end{aligned}
$$

and so Lemma 2.4 yields that $\mu$ is geometric $\alpha$-Carleson.

\section{4. $L^{p}$-ADMISSIBLE CONTROL OPERATORS}

We now consider the case that $U=X=\ell_{q}$. Let $B: U \rightarrow X_{-1}$ be linear and bounded. Then there are functionals $\varphi_{n} \in\left(\ell_{q}\right)^{*}$ such that $(B u)_{n}=\left\langle\varphi_{n}, u\right\rangle$. Indeed, $B$ is determined by its values on any basis $\left(e_{j}\right)$ of $U$. We choose $\left(e_{n}\right)$ to be the standard basis in $\ell_{q}$. Let $\varphi_{n}$ be the sequence $\left(\left\langle e_{n}, B e_{j}\right\rangle\right)_{j=1}^{\infty}$, i.e. $\varphi_{n}$ is the scalar sequence of $n^{\text {th }}$ coordinates of the vector-valued sequence $\left(B e_{j}\right)$. Then, for $u=\left(u_{j}\right) \in U$, and $n \in \mathbb{N}$,

$$
\left\langle e_{n}, B u\right\rangle=\left\langle e_{n}, \sum u_{j} B e_{j}\right\rangle=\sum u_{j}\left\langle e_{n}, B e_{j}\right\rangle
$$

is a finite number whence $\varphi_{n} \in\left(\ell_{q}\right)^{*}$ for all $n$. So, $\left\langle e_{n}, B u\right\rangle=\left\langle\varphi_{n}, u\right\rangle$, which proves the claim. This means that any bounded linear operator $B: U \rightarrow X_{-1}$ may be interpreted as a certain sequence of functionals. The following proposition is a direct generalisation of [29, Proposition 4.8.6].

Proposition 4.1. Let $X=U=\ell_{q}$ and $1<p \leq q<\infty$. Let $\left(\varphi_{n}\right)$ be a sequence of elements in $U^{*}=\ell_{q^{\prime}}$ and consider the scalar sequence $b$ defined by $b_{n}=\left\|\varphi_{n}\right\|$ and let the operator $B$ defined by $(B u)_{n}=\left\langle\varphi_{n}, u\right\rangle$.

Then, if $b$ is an $L^{p}$-admissible input element for $A, B$ is bounded from $U$ to $X_{-\theta}$ for all $\theta>1 / p^{\prime}$ and $B$ is an $L^{p-a d m i s s i b l e ~ c o n t r o l ~ o p e r a t o r ~ f o r ~} A$.

Proof. From the proof of Theorem 3.1, we know that $b$ is an element of $X_{-\theta}$ for all $\theta>1 / p^{\prime}$. The elementary estimate $\left|\left\langle\varphi_{n}, u\right\rangle\right| \leq\|u\|\left\|\varphi_{n}\right\|$ then implies that $B$ is linear and bounded from $U$ to $X_{-\theta}$. Now let $u \in L^{p}(0, \infty ; U)=L^{p}\left(0, \infty, \ell_{q}\right)$ and let $u_{j}(\cdot)$ denote its coordinate functions. Then

$$
\left\|\int_{0}^{t} S(t-s) B u(s) d s\right\|_{\ell_{q}}=\left(\sum_{n=1}^{\infty}\left|\int_{0}^{t} e^{-\lambda_{n}(t-s)}\left\langle\varphi_{n}, u(s)\right\rangle d s\right|^{q}\right)^{1 / q}
$$




$$
\begin{aligned}
& =\left(\sum_{n=1}^{\infty}\left|\left\langle\varphi_{n}, \int_{0}^{t} e^{-\lambda_{n}(t-s)} u(s)\right\rangle d s\right|^{q}\right)^{1 / q} \\
& \leq\left(\sum_{n=1}^{\infty}\left\|\varphi_{n}\right\|^{q}\left\|\int_{0}^{t} e^{-\lambda_{n}(t-s)} u(s) d s\right\|_{U}^{q}\right)^{1 / q} \\
& =\left(\sum_{n=1}^{\infty}\left\|\varphi_{n}\right\|^{q} \sum_{j=1}^{\infty}\left|\int_{0}^{t} e^{-\lambda_{n}(t-s)} u_{j}(s) d s\right|^{q}\right)^{1 / q} \\
& =\left(\sum_{j=1}^{\infty}\left[\sum_{n=1}^{\infty}\left|\int_{0}^{t} e^{-\lambda_{n}(t-s)}\left\|\varphi_{n}\right\| u_{j}(s) d s\right|^{q}\right]\right)^{1 / q} .
\end{aligned}
$$

Now, by assumption, we may estimate

$$
\begin{aligned}
& \leq C\left(\sum_{j=1}^{\infty}\left\|u_{j}\right\|_{L^{p}}^{q}\right)^{1 / q}=C\|u\|_{\ell_{q}\left(L^{p}(0, \infty)\right)} \\
& \leq C\|u\|_{L^{p}(0, \infty ; U)}
\end{aligned}
$$

where the last estimate (namely that $\|f\|_{\ell_{q}\left(L^{p}\right)} \leq\|f\|_{L^{p}\left(\ell_{q}\right)}$ if $p \leq q$ ) is a easy corollary of Minkowski's inequality, see e.g. [6, Exercise VI.11.14].

Notice that combining the Proposition with Theorems 3.1 and 3.2 yields a sufficient condition for $L^{p}$-admissibility for control operators $B: U \rightarrow X_{-1}$ via a Carleson measure criterion under suitable conditions on $p$ and $q$.

\section{5. $L^{p}-$ ADMISSIBILITY OF RECIPROCAL SYSTEMS}

Suppose that $\alpha=p^{\prime} / q \leq 1$ and $p \leq 2$. Then, if $b$ is $L^{p^{\prime}}$-admissible for $A$, Theorem 3.2 tell us that the discrete measure $\mu=\sum_{n}\left|\frac{b_{n}}{\lambda_{n}}\right|^{q} \delta_{\lambda_{n}^{-1}}$ is embedding $\alpha^{-}$ Carleson. Since the numbers $\frac{b_{n}}{\lambda_{n}}$ are the coefficients of $A^{-1} b$ and since the support of the measure is equal to the set of eigenvalues of $A^{-1}$, Theorem 3.1 implies that $A^{-1} b$ is an $L^{p}$ admissible input element for $A^{-1}$.

This observation can be formalised in the following question: given a sectorial operator $A$ and a (control) operator $B \in \mathcal{B}\left(U, X_{-1}\right)$, does $L^{p^{\prime}}$-admissibility of the pair $A, B$ imply the $L^{p}$-admissibility of the pair $A^{-1}, A^{-1} B$ ? This question is not new. In fact, the system

$$
z^{\prime}(t)+A^{-1} z(t)=A^{-1} B u(t), \quad z(0)=z_{0} .
$$

is called the associated reciprocal linear system to (1.1). By considering Lyapunov equations CURTAIN shows in the case of Hilbert spaces $X$ and $U$ that, under the assumption that $0 \in \varrho(A)$, an operator $B$ is infinite-time $L^{2}$-admissible for $A$ if and only if $A^{-1} B$ is infinite-time $L^{2}$-admissible for $A^{-1}$ (see [5, Theorem 5]). We shall discuss generalisations of this result below.

Analytic semigroups on Banach spaces. The famous Weiss conjecture (see [36]) states that $L^{2}$-admissibility of a control operator $B$ between Hilbert spaces $U$ and $X$ is characterised by the boundedness of the set

$$
\left\{\operatorname{Re}(\lambda)^{1 / 2}(\lambda+A)^{-1} B: \operatorname{Re}(\lambda)>0\right\}
$$

in $\mathcal{B}(U, X)$. The conjecture holds for normal semigroups and $U=\mathbb{C}$ but fails even in case of $U=\mathbb{C}$ for general semigroups on Hilbert spaces (see [18]). For a survey on positive and negative results concerning the Weiss conjecture we refer to [17.

For analytic semigroups, $L^{2}$-admissibility is characterised by (5.2) even in Banach space under an inevitable additional condition (see [19]). This characterisation 
has subsequently been generalised to $L^{p}$-norms with certain weights (see [13] and [12]). We briefly summarise some required notions and results:

For sectorial operators $A$ there exists a natural functional calculus on the algebra of functions

$$
H_{0}^{\infty}(S(\omega))=\left\{f \in H^{\infty}(S(\omega)): \exists c, s>0:|f(z)| \leq c \min \left(|z|^{s},|z|^{-s}\right)\right\}
$$

that is given by $f(A):=\int_{\Gamma} f(z) R(z, A) d z$. Here, $\Gamma$ is the positively orientated boundary of a sector $S(\theta)$ containing the spectrum of $A$ (see [14, 20, for more details). Since $B=A^{1 / 2}$ always satisfies condition (5.2), we find that whenever the Weiss conjecture holds true, one has

$$
\int_{0}^{\infty}\|\varphi(t A) x\|^{2} \frac{d t}{t}=\int_{0}^{\infty}\left\|(t A)^{1 / 2} S(t) x\right\|^{2} \frac{d t}{t} \leq m^{2}\|x\|^{2}
$$

for $\varphi(z)=z^{1 / 2} \exp (-z)$. By a result of $\mathrm{M}^{c}$ InTOSH and YAGI [21, Theorem 5], inequality (5.3) does not depend on the particular choice of the function $\varphi \in$ $H_{0}^{\infty}(S(\omega)) \backslash\{0\}$. This result extends to Banach spaces and $L^{p_{-}}$norms for all $p \in$ $[1, \infty]$. Such estimates are called $L_{*}^{p}$-estimates, where the subscript refers to the measure $d t / t$ on $\mathbb{R}_{+}$. They automatically hold for $p=\infty$. Moreover, $L_{*}^{p}$ estimates for $A$ imply $L_{*}^{q}$-estimates for all $q \geq p$ (see [12, Remark 1.6]). This is due to the fact that $L_{*}^{p}$-estimates for $A$ are equivalent to $\left.X \hookrightarrow\left(\dot{X}_{-1}, \dot{X}_{1}\right)\right)_{1 / 2, p}$ whereas $L_{*}^{q}$-estimates for $A^{\prime}$ are equivalent to $\left(\dot{X}_{-1}, \dot{X}_{1}\right)_{1 / 2, q^{\prime}} \hookrightarrow X$. We quote a characterisation of admissibility for control operators from [12]. Recall that a sectorial operator with dense range is actually injective (see [21, Thm. 3.8]).

Theorem 5.1. Let $p \in[1, \infty)$ and $A$ be a densely defined sectorial operator of type $\omega<\pi / 2$ with dense range on $X$. For $B \in \mathcal{B}\left(U, X_{-1}\right)$ consider the set

$$
W_{A, B}^{(p)}:=\left\{\lambda^{1 / p}(\lambda+A)^{-1} B: \lambda>0\right\} \subseteq \mathcal{B}(U, X) .
$$

Then the following assertions hold:

(a) If $B$ is $L^{p}$-admissible for $A$, then $W_{A, B}^{(p)}$ is bounded.

(b) If $W_{A, B}^{(p)}$ is bounded and the adjoint operator $A^{\prime}$ satisfies $L_{*}^{p^{\prime}}$-estimates then $B$ is $L^{p}$-admissible for $A$.

As in the case $p=2$, the additional condition in (b) is optimal.

Corollary 5.2. Let $p \in[1, \infty)$ and $A$ be a densely defined sectorial operator of type $\omega<\pi / 2$ with dense range on $X$ and let $B \in \mathcal{B}\left(U, X_{-1}\right)$.

(a) If $B$ is $L^{p}$-admissible for $A$ and if $A^{\prime}$ satisfies $L_{*}^{p}$-estimates on $X^{\prime}$, then $A^{-1} B$ is $L^{p^{\prime}}-$ admissible for $A^{-1}$.

(b) If $A^{-1} B$ is $L^{p^{\prime}}-$ admissible for $A^{-1}$ and if $A^{\prime}$ satisfies $L_{*}^{p^{\prime}}$-estimates on $X^{\prime}$, then $B$ is $L^{p}$-admissible for $A$.

Proof. Notice that whenever $\varphi \in H_{0}^{\infty}(S(\theta))$, the function $\psi(z)=\varphi(1 / z) \in H_{0}^{\infty}(S(\theta))$. By functional calculus, $\varphi(t A)=\psi\left(t^{-1} A^{-1}\right.$ ) (see [14, Proposition 2.4.1]) and thus $A$ admits $L_{*}^{r}$-estimates if, and only if its inverse $A^{-1}$ does. In view of Theorem 5.1 it is therefore sufficient to consider the expressions

$$
\lambda^{1 / p}(\lambda+A)^{-1} B=\lambda^{1 / p-1}\left(\lambda^{-1}+A^{-1}\right)^{-1} A^{-1} B=z^{1 / p^{\prime}}\left(z+A^{-1}\right)^{-1} A^{-1} B
$$

where $\lambda^{-1}=z$. Now, if $B$ is $L^{p}$ admissible, $W_{A, B}^{(p)}$ is bounded, whence $W_{A^{-1}, A^{-1} B}^{\left(p^{\prime}\right)}$ is bounded. If $A^{\prime}$ satisfies $L_{*}^{p}$-estimates, then $A^{-1} B$ is $L^{p^{\prime}}$-admissible for $A$. For the converse we need to impose $L_{*}^{p^{\prime}}$-estimates on $A^{\prime}$ by the same argument. 
Remark 5.3. We can use the corollary to compare Theorems 3.1 and 3.2 in the following case: let $A$ generate a bounded analytic semigroup and assume that

$$
\mu=\sum_{n=1}^{\infty} \delta_{\lambda_{n}}\left|b_{n}\right|^{q}=\sum_{n=1}^{\infty} \delta_{\left(\lambda_{n}^{-1}\right)^{-1}}\left|\frac{\lambda_{n}^{-1} b_{n}}{\lambda_{n}^{-1}}\right|^{q}
$$

is embedding $\alpha$-Carleson with $\alpha={ }^{p^{\prime}} / q$, so that Theorem 3.2 yields $L^{p^{\prime}}$-admissibility of $A^{-1} b$ for $A^{-1}$. If $p \leq q$ Corollary [5.2 implies that $b$ is $L^{p}$-admissible for $A$ since in this case the Lorentz space $\ell_{q, p}$ embeds into $\ell_{q, q}=\ell_{q}$. Both theorems coincide therefore in this case and it is remarkable, that the restriction $p \leq 2$ in Theorem 3.1 may be weakened to $p \leq q$ if $q>2$, thus allowing in particular the natural choice $p=q$ for all $p \in(1, \infty)$. In view of Example 2.5 (e) analyticity of the semigroup seems to be a necessary restriction to guarantee this improvement of Theorem 3.1

If, conversely, $b$ is $L^{p}$-admissible for $A$, Corollary 5.2 guarantees the $L^{p^{\prime}}$-admissibility of $A^{-1} b$ for $A^{-1}$ when $p^{\prime} \leq q$ and Theorem 3.2 yields that $\mu$ is geometric $\alpha$-Carleson, so both theorems again coincide.

Arbitrary semigroups on Banach spaces. In the sequel we want to get rid of the assumption of analyticity and give a more direct argument for passing

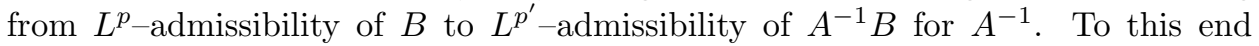
we assume that $S(t)=\exp (-t A)$ is uniformly exponentially stable. Then, by the Phillips functional calculus (see e.g. [14]) one obtains different representation formulas for $\exp \left(-t A^{-1}\right)$ that may turn out to be useful for different problems. The most interesting (to us) are

$$
\exp \left(-t A^{-1}\right) x=x-\int_{0}^{\infty}\left(\frac{t}{s}\right)^{1 / 2} J_{1}(2 \sqrt{s t}) S(s) x d s
$$

for $x \in X$ where $J_{1}$ is the first Bessel function of first order. This formula was given in [11, 10, 39, 38. It is an immediate consequence of [24, Formula (5.67)]. Moreover we have

$$
\begin{gathered}
A^{-1 / 2} \exp \left(-t A^{-1}\right) x=\int_{0}^{\infty} \frac{1}{\sqrt{\pi s}} \cos (2 \sqrt{s t}) S(s) x d s \\
A^{-\nu-1} \exp \left(-t A^{-1}\right) x=\int_{0}^{\infty}\left(\frac{t}{s}\right)^{\nu / 2} I_{\nu}(2 \sqrt{s t}) S(s) x d s, \quad \nu>-1
\end{gathered}
$$

for $x \in X$ as a consequence of [24, Formulas (5.70) and (5.75)]. Here, $I_{\nu}$ is the modified Bessel function of the first kind, of order $\nu$ (see e.g. [1, 32 for more details on Bessel functions).

Since for general exponentially stable semigroups, the decay rate

$$
\left\|\exp \left(-t A^{-1}\right) A^{-1} x\right\|=O\left((1+t)^{-1 / 4}\right)\|x\|
$$

is optimal (see [39, Theorem 3.3 and Example 3.5], one cannot expect more than that $L^{p}$-admissibility of $B$ implies finite-time $L^{p^{\prime}}$-admissibility of $A^{-1} B$ for $A^{-1}$. In this sense the following result is optimal.

Theorem 5.4. Let $-A$ generate an exponentially stable semigroup $S(t)_{t \geq 0}$ on a Banach space $X$, suppose that $A$ has dense range and that $B \in \mathcal{B}\left(U, X_{-1}\right)$ is $L^{p_{-}}$ admissible for $A, p \in[1, \infty]$. Then $A^{-1} B$ is finite-time $L^{p^{\prime}}$-admissible for $A^{-1}$.

Proof. Let $T>0$. Then we may find $\varepsilon>0$ such that $B$ is $L^{p}$-admissible for the shifted semigroup $e^{+\varepsilon t} S(t)$ as well. By formula (5.7) with $\nu=0$ we obtain

$$
\left\|\int_{0}^{T} \exp \left(-s A^{-1}\right) A^{-1} B u(s) d s\right\|_{X}
$$




$$
\begin{aligned}
& =\left\|\int_{0}^{T}\left(\int_{0}^{\infty} I_{0}(2 \sqrt{s t}) S(t) d t\right) B u(s) d s\right\|_{X} \\
& =\left\|\int_{0}^{\infty} e^{+\varepsilon t} S(t) B\left(e^{-\varepsilon t} \int_{0}^{T} I_{0}(2 \sqrt{s t}) u(s) d s\right) d t\right\|_{X} \\
& \leq M\left\|e^{-\varepsilon t} \int_{0}^{T} I_{0}(2 \sqrt{s t}) u(s) d s\right\|_{L^{p}((0, \infty), U)} \\
& \leq M\left\|e^{-\varepsilon t} \int_{0}^{T}\left|I_{0}(2 \sqrt{s t})\right|\right\| u(s)\|d s\|_{L^{p}(0, \infty)}\|\|_{L^{p^{\prime}(0, T)}}\left\|t \mapsto e^{-\varepsilon t}\right\| s \mapsto I_{0}(2 \sqrt{s t})\left\|_{L^{p}(0, T)}\right\|_{L^{p}(0, \infty)}=: M C_{T}\|u\|_{L^{p^{\prime}(0, T)}} .
\end{aligned}
$$

From [1, Formula (9.6.16)], we deduce the following integral representation

$$
I_{0}(x)=\frac{1}{\pi} \int_{0}^{\pi} e^{x \cos (\theta)} d \theta=\frac{2}{\pi} e^{x} \int_{0}^{1} \exp \left(-2 u^{2} x\right)\left(1-u^{2}\right)^{-1 / 2} d u
$$

by letting $u=\sin (\theta / 2)$ (see also [32, p. 204]). In particular, $\left|I_{0}(x)\right| \leq e^{x}, x>0$. In the case $p=\infty$ boundedness of $C_{T}$ is immediate. In case the $p<\infty$,

$$
f(t):=\left\|s \mapsto I_{0}(2 \sqrt{s t})\right\|_{L^{p}(0, T)}^{p} \leq \frac{2}{t p^{2}}\left(1+e^{p \sqrt{t T}}(p \sqrt{t T}-1)\right) .
$$

Now, since $\lim _{t \rightarrow 0} f(t)=T$ it is clear that $e^{-\varepsilon t} f(t)$ is integrable on $(0, \infty)$ for any $\varepsilon>0$. Thus $C_{T}<\infty$ and so $A^{-1} B$ is finite-time $L^{p^{\prime}}$-admissible for $A^{-1}$.

Acknowledgement. We are indepted to Birgit Jacob, Peer Christian Kunstmann and Elizabeth Strouse for helpful remarks and discussions.

\section{REFERENCES}

[1] Milton Abramowitz and Irene A. Stegun, Handbook of Mathematical Functions with Formulas, Graphs, and Mathematical Tables, National Bureau of Standards Applied Mathematics Series, vol. 55, For sale by the Superintendent of Documents, U.S. Government Printing Office, Washington, D.C., 1964.

[2] Éric Amar and Aline Bonami, Mesures de Carleson d'ordre $\alpha$ et solutions au bord de l'équation $\bar{\partial}$, Bull. Soc. Math. France 107 (1979), no. 1, 23-48.

[3] Lennart Carleson, An interpolation problem for bounded analytic functions, Amer. J. Math. 80 (1958), 921-930.

[4] Interpolations by bounded analytic functions and the corona problem, Ann. of Math. (2) 76 (1962), 547-559.

[5] Ruth F. Curtain, Regular linear systems and their reciprocals: applications to Riccati equations, Systems Control Lett. 49 (2003), no. 2, 81-89.

[6] Nelson Dunford and Jacob T. Schwartz, Linear operators. Part I, Wiley Classics Library, John Wiley \& Sons Inc., New York, 1988, General theory, With the assistance of William G. Bade and Robert G. Bartle, Reprint of the 1958 original, A Wiley-Interscience Publication.

[7] Peter L. Duren, Extension of a theorem of Carleson, Bull. Amer. Math. Soc. 75 (1969), $143-146$.

[8] Klaus-Jochen Engel and Rainer Nagel, One-parameter semigroups for linear evolution equations, Graduate Texts in Mathematics, vol. 194, Springer-Verlag, New York, 2000, With contributions by S. Brendle, M. Campiti, T. Hahn, G. Metafune, G. Nickel, D. Pallara, C. Perazzoli, A. Rhandi, S. Romanelli and R. Schnaubelt.

[9] C. Fefferman and E. M. Stein, Some maximal inequalities, Amer. J. Math. 93 (1971), 107115.

[10] A. M. Gomilko, Kh. Zvart, and Yu. Tomilov, On the inverse operator of the generator of a $C_{0}$-semigroup, Mat. Sb. 198 (2007), no. 8, 35-50.

[11] Alexander Gomilko and Hans Zwart, The Cayley transform of the generator of a bounded $C_{0}$-semigroup, Semigroup Forum 74 (2007), no. 1, 140-148.

[12] Bernhard H. Haak and Peer Chr. Kunstmann, Weighted admissibility and wellposedness of linear systems in Banach spaces, SIAM J. Control Optim. 45 (2007), no. 6, 2094-2118.

[13] Bernhard H. Haak and Christian Le Merdy, $\alpha$-admissibility of observation and control operators, Houston J. Math. 31 (2005), no. 4, 1153-1167. 
[14] Markus Haase, The functional calculus for sectorial operators, Operator Theory, Advances and Applications, vol. 169, Birkhäuser Verlag, 2006

[15] L. F. Ho and D. L. Russell, Admissible input elements for systems in Hilbert space and a Carleson measure criterion, SIAM J. Control Optim. 21 (1983), no. 4, 614-640, Erratum in the same journal, Vol. 21,No. 6, p. 985-986.

[16] Birgit Jacob, Jonathan Partington, and Sandra Pott, Tangential Interpolation in Weighted Vector-valued $H^{p}$ Spaces, with Applications, To appear in Complex Analysis and Operator Theory, http://dx.doi.org/10.1007/s11785-008-0081-y

[17] Birgit Jacob and Jonathan R. Partington, Admissibility of control and observation operators for semigroups: a survey, Current trends in operator theory and its applications, Oper. Theory Adv. Appl., vol. 149, Birkhäuser, Basel, 2004, pp. 199-221.

[18] Birgit Jacob and Hans Zwart, Counterexamples concerning observation operators for $C_{0}$ semigroups, SIAM J. Control Optim. 43 (2004), no. 1, 137-153.

[19] Christian Le Merdy, The Weiss conjecture for bounded analytic semigroups, J. London Math. Soc. (2) 67 (2003), no. 3, 715-738.

[20] Alan McIntosh, Operators which have an $H_{\infty}$ functional calculus, Miniconference on operator theory and partial differential equations (North Ryde, 1986), Proc. Centre Math. Anal. Austral. Nat. Univ., vol. 14, Austral. Nat. Univ., Canberra, 1986, pp. 210-231.

[21] Alan McIntosh and Atsushi Yagi, Operators of type $\omega$ without a bounded $H_{\infty}$ functional calculus, Miniconference on Operators in Analysis (Sydney, 1989), Proc. Centre Math. Anal. Austral. Nat. Univ., vol. 24, Austral. Nat. Univ., Canberra, 1990, pp. 159-172.

[22] J. Darrell McPhail, A weighted interpolation problem for analytic functions, Studia Math. 96 (1990), no. 2, 105-116.

[23] Takahiko Nakazi, Interpolation of weighted $l^{q}$ sequences by $H^{p}$ functions, Taiwanese J. Math. 9 (2005), no. 3, 457-467.

[24] Fritz Oberhettinger and Larry Badii, Tables of Laplace transforms, Springer-Verlag, New York, 1973.

[25] Dietmar Salamon, Infinite-dimensional linear systems with unbounded control and observation: a functional analytic approach, Trans. Amer. Math. Soc. 300 (1987), no. 2, 383-431.

[26] Elias M. Stein, Singular Integrals and Differentiability Properties of Functions, Princeton Mathematical Series, No. 30, Princeton University Press, Princeton, N.J., 1970.

[27] _ Harmonic Analysis: Real-variable Methods, Orthogonality, and Oscillatory Integrals, Princeton Mathematical Series, vol. 43, Princeton University Press, Princeton, NJ, 1993, With the assistance of Timothy S. Murphy, Monographs in Harmonic Analysis, III.

[28] B. A. Taylor and D. L. Williams, Interpolation of $l^{q}$ sequences by $H^{p}$ functions, Proc. Amer. Math. Soc. 34 (1972), 181-186.

[29] Marius Tucsnak and George Weiss, Observation and Control for Operator Semigroups, Birkhäuser Verlag, Basel, 2009.

[30] Michael Unteregge, $p$-admissible control elements for diagonal semigroups on $l^{r}$-spaces, Systems Control Lett. 56 (2007), no. 6, 447-451.

[31] I. V. Videnskiı̌, An analogue of Carleson measures, Dokl. Akad. Nauk SSSR 298 (1988), no. $5,1042-1047$.

[32] G. N. Watson, A treatise on the theory of Bessel functions, Cambridge Mathematical Library, Cambridge University Press, Cambridge, 1966, Reprint of the second (1944) edition.

[33] George Weiss, Admissibility of input elements for diagonal semigroups on $l^{2}$, Systems Control Lett. 10 (1988), no. 1, 79-82.

[34] _ Admissibility of unbounded control operators, SIAM J. Control Optim. 27 (1989), no. $3,527-545$.

[35] — Admissible observation operators for linear semigroups, Israel J. Math. 65 (1989), no. $1,17-43$.

[36] _ Two conjectures on the admissibility of control operators, Estimation and control of distributed parameter systems (Vorau, 1990), Internat. Ser. Numer. Math., vol. 100, Birkhäuser, Basel, 1991, pp. 367-378.

[37] Andrew Wynn, $\alpha$-admissibility of observation operators in discrete and continuous time, To appear in Complex Analysis and Operator Theory.

[38] Hans Zwart, Growth estimates for $\exp \left(A^{-1} t\right)$ on a Hilbert space, Semigroup Forum 74 (2007), no. 3, 487-494.

[39] Is $^{-1}$ an infinitesimal generator?, Perspectives in operator theory, Banach Center Publ., vol. 75, Polish Acad. Sci., Warsaw, 2007, pp. 303-313.

Institut de Mathématiques de Bordeaux, Université Bordeaux 1, 351, Cours de la Libération, 33405 TAlence CEDEX, FRANCE

E-mail address: bernhard.haak@math.u-bordeaux1.fr 